\title{
Translational Control via the Mammalian Target of Rapamycin Pathway Is Critical for the Formation and Stability of Long-Term Fear Memory in Amygdala Neurons
}

\author{
Ryan G. Parsons, ${ }^{\star}$ Georgette M. Gafford, ${ }^{\star}$ and Fred J. Helmstetter \\ Department of Psychology, University of Wisconsin-Milwaukee, Milwaukee, Wisconsin 53201
}

\begin{abstract}
The mammalian target of rapamycin kinase (mTOR) regulates protein synthesis in neurons at the translational level through phosphorylation of several intracellular targets. Recent work in invertebrates indicates that mTOR-dependent translational control may be critical for the induction and maintenance of activity-dependent synaptic plasticity underlying memory formation. Here, we report that training rats in a simple fear conditioning procedure evokes a time-dependent increase in the phosphorylation of p70s6 kinase, a major direct downstream target of mTOR. When the activation of mTOR was prevented by posttraining injection of rapamycin into the amygdala, formation of the memory and the increase in p70s6 kinase phosphorylation was attenuated. Furthermore, when rapamycin was applied to the amygdala after the recall of a previously stored fear memory, subsequent retention was disrupted, indicating that local translational control at active synapses is required for the stability as well as the formation of long-term memory in this system.
\end{abstract}

Key words: reconsolidation; consolidation; local protein synthesis; translational control; synaptic plasticity; fear conditioning

\section{Introduction}

The signaling pathway controlled by the mammalian target of rapamycin (mTOR) kinase regulates protein translation by controlling the phosphorylation state of the eIF4E-binding protein 1 (4E-BP1) and p70s6 kinase (p70s6K) (for review, see Raught et al., 2001). Rapamycin (RAP) is an inhibitor of the mTOR pathway, and work in various preparations has pointed to this pathway as being critical for different types of synaptic plasticity (Casadio et al., 1999; Tang et al., 2002; Cammalleri et al., 2003). Several reports point to a role for the mTOR pathway in synaptic plasticity, but few studies have examined the role of mTOR in memory formation (Tischmeyer et al., 2003).

The amygdala is thought to be the brain area where critical synaptic plasticity occurs, supporting the formation of fear memory (Fanselow and LeDoux, 1999). Previous research has shown that the consolidation of fear memory in the amygdala depends on macromolecular synthesis and various other specific intracellular mechanisms (Bailey et al., 1999; Schafe and LeDoux, 2000; Schafe et al., 2000; Josselyn et al., 2001). Despite the progress in understanding how the amygdala consolidates fear memory, very little is known about the role of translational regulation in fear memory and in memory formation in general.

A few recent studies have suggested that translational control is a critical feature of synaptic plasticity and memory formation.

\footnotetext{
Received Sept. 26, 2006; revised Nov. 3, 2006; accepted Nov. 4, 2006.

This work was supported by National Institute of Mental Health Grants MH069558 (F.J.H.) and MH071126 (R.G.P.).

*R.G.P. and G.M.G. contributed equally to this work.

Correspondence should be addressed to Fred J. Helmstetter, Department of Psychology, University of WisconsinMilwaukee, Garland Hall, P.0. Box 413, Milwaukee, WI 53201. E-mail: fjh@uwm.edu.

DOI:10.1523/JNEUROSCI.4209-06.2006

Copyright $\odot 2006$ Society for Neuroscience $\quad$ 0270-6474/06/2612977-07\$15.00/0
}

Kelleher et al. (2004) demonstrated that the extracellular signalregulated kinase (ERK)/mitogen-activated protein kinase pathway is involved in regulating the phosphorylation state of numerous factors implicated in controlling translation initiation, including eIF4E, 4E-BP1, and the ribosomal protein S6. The genetically altered mice used in this study also showed deficits in long-term potentiation (LTP) and hippocampus-dependent memory. Other recent studies using mice knock-out models have also argued for a role of translational regulation in synaptic plasticity and memory (Banko et al., 2005; Costa-Mattioli et al., 2005).

Although a handful of studies have argued for a role of translational regulation in memory formation and neuronal plasticity, these studies primarily used genetic mouse models, which often lack temporal and brain region specificity. These recent studies have also focused almost exclusively on the hippocampus, whereas very little work has examined the involvement of translational regulation in hippocampus-independent memories. Furthermore, no published studies have determined whether this mechanism is involved in the reconsolidation of memory after retrieval, which refers to the idea that memories become labile after reactivation whereby protein synthesis is needed to restabilize them (Nader et al., 2000).

In the current study, we gave infusions of RAP into the amygdala immediately after fear conditioning to determine whether disruption of the mTOR pathway in the amygdala affects fear memory formation. Separate experiments sought to characterize the expression of total and phospho-p70s6K in amygdala homogenates in time periods after fear conditioning and treatment with RAP. Finally, we examined the effect of RAP treatment on fear memory reconsolidation in the amygdala. These experiments provide evidence that translational events controlled by the 
mTOR pathway contribute to long-term fear memory formation in the amygdala.

\section{Materials and Methods}

Subjects. One hundred fifty-four naive male Long Evans rats obtained from Harlan (Madison, WI) weighing 300-350 g served as subjects. All animals were individually housed in stainless-steel hanging cages and housed in a room maintained on a 14:10 h light/dark cycle. Experiments took place during the light portion of the cycle. Food and water were available ad libitum. All procedures were performed with approval from the Institutional Animal Care and Use Committee.

Surgery. Animals were handled for several days before surgery. Rats that underwent surgery were implanted with bilateral cannulas aimed at the amygdala [anteroposterior (AP), -2.8 ; lateral (L), \pm 5.0 ; ventral $(\mathrm{V})$, -7.2 . Coordinates were chosen based on a rat brain atlas (Paxinos and Watson, 1998). Before surgery, animals were anesthetized with intraperitoneal injections of ketamine $\mathrm{HCl}(100 \mathrm{mg} / \mathrm{kg}$ body weight $)$ and sodium pentobarbital $(2.5 \mathrm{mg} / \mathrm{kg} / \mathrm{rat})$. The cannulas were anchored to the skull using stainless-steel screws and acrylic cement. Obdurators (33 gauge) were inserted into the guide cannulas to prevent blockage.

Apparatus. Auditory fear conditioning was conducted in a set of four Plexiglas and stainless-steel observation chambers (Context A) housed in sound-attenuating chambers. The floor was comprised of 18 stainlesssteel bars $5 \mathrm{~mm}$ in diameter spaced $12 \mathrm{~mm}$ apart and connected to a shock generator. Ventilation fans produced $62-64 \mathrm{~dB}$ of background noise. Each chamber was equipped with a speaker centered in the middle of one end of the chamber. Before testing of each animal, Context A was cleaned with a 5\% ammonium hydroxide solution.

Fear to the auditory conditioned stimulus (CS) was tested in chambers (Context B) that had slanted walls on one side and a rounded wall on the other side. The floors were made of Plexiglas, and fans provided a background noise of $\sim 58 \mathrm{~dB}$. Each chamber was enclosed in a soundattenuating box and illuminated with a white light. Before testing of each rat, the chambers in Context B were wiped down with a $2 \%$ acetic acid solution.

Drug preparation and infusion procedure. In all cases, rats received bilateral infusions into the amygdala. The total volume of the infusion $(0.5 \mu \mathrm{l} / \mathrm{side})$ was given over $60 \mathrm{~s}$, and the injection cannula remained in place for an additional $90 \mathrm{~s}$ to ensure diffusion away from the injector tip. The injection cannulas were cut to extend $\sim 0.5 \mathrm{~mm}$ beyond the guide cannulas. Rats were returned to their home cages after infusions. Rapamycin (Calbiochem, La Jolla, CA) was dissolved in 100\% Dimethyl Sulfoxide (Sigma, St. Louis, MO) to the appropriate dose.

Behavioral procedures. One week after surgery, animals were habituated to the handling and injection procedure. To habituate the animal to the microinjection procedure, each rat was restrained in a towel for several minutes, the obdurators were removed, and the scalp was cleaned. During this time, the infusion pump to be used during the experiment was activated to habituate the animals to the sound it produces. After this was complete, the obdurators were replaced and the animal was returned to its home cage. This was repeated once a day for $4 \mathrm{~d}$.

Training involved a 6 min baseline followed by four white noise (72 $\mathrm{dB} ; 10 \mathrm{~s}$ )-shock ( $1 \mathrm{~mA} / 1 \mathrm{~s}$ for initial dose-response experiment) (1.3 $\mathrm{mA} / 1 \mathrm{~s}$ for experiment 2 in Fig. $1 c, d)$ pairings separated by a $90 \mathrm{~s}$ intertrial interval. After a 4 min postshock period, animals were removed from the training context (Context A). Immediately after the training session, rats were brought into an adjacent room and given infusions of RAP or DMSO. In the first experiment, rats $(n=38)$ were given infusions of DMSO or different doses $(5,50,100,500,1000 \mathrm{ng} / \mu \mathrm{l})$ of RAP into the amygdala. In the second experiment, the animals $(n=23)$ were given $\mathrm{RAP}(5 \mu \mathrm{g} / \mu \mathrm{l})$ or DMSO immediately after training. Approximately $24 \mathrm{~h}$ later, animals were placed into Context $\mathrm{A}$ for a context test as well as a novel context never paired with shock (Context B) in a counterbalanced order. The context test involved exposing animals to Context A for 15 min. During the auditory CS test, animals were placed in Context B for 15 $\mathrm{min}$ in which a $6 \mathrm{~min}$ baseline was followed by $5 \mathrm{~min}$ of continuous exposure to the white noise. Rats were removed from the chamber $4 \mathrm{~min}$ after the white noise ended and taken to their home cage.

Training for the immediate versus delayed shock experiment went as follows. Animals in the immediate shock group $(n=8)$ were placed into Context A, immediately shocked $(1.3 \mathrm{~mA} ; 2 \mathrm{~s})$, and allowed to remain in the chamber for $3 \mathrm{~min}$. The delay group $(n=9)$ was allowed to explore Context A for $2 \mathrm{~min}$, received a shock $(1.3 \mathrm{~mA} ; 2 \mathrm{~s})$, and remained in context $\mathrm{A}$ for another minute. Both immediate and delay animals were killed 60 min after training. A third group, the home cage control $(n=8)$ was killed directly from their cage. Animals were processed for Western blot $(n=22)$ or immunofluorescence $(n=3)$.

The training procedure for the reconsolidation experiment was exactly the same as in experiment 1 . To reactivate the memory, rats were brought into Context $\mathrm{B}$ the following day and exposed to a $32 \mathrm{~s}$ presentation of the white noise after a $6 \mathrm{~min}$ baseline period. Rats were removed from the chamber and brought into an adjacent room where they received infusions of RAP $(1 \mu \mathrm{g} / \mu \mathrm{l} ; n=12)$ or DMSO $(n=11)$ into the amygdala. Twenty-four hours later, the rats were tested to the white noise for $2 \mathrm{~min}$ after a 6 min baseline.

After completion of testing, animals were killed with an intraperitoneal injection of sodium pentobarbital $(100 \mathrm{mg} / \mathrm{kg})$. Animals were transcardially perfused with saline followed by $10 \%$ buffered formalin solution. Heads, with cannulas intact, were placed in $10 \%$ formalin solution for at least $24 \mathrm{~h}$. The brains were then extracted from the skull and placed in a $30 \%$ sucrose formalin solution until they were ready to section. Frozen sections $(40 \mu \mathrm{m})$ were collected throughout the amygdala, mounted on slides, and stained with cresyl violet. Injection sites were then determined with the aid of a rat brain atlas (Paxinos and Watson, 1998).

Western blot experiments. Rats were exposed to the standard fearconditioning procedure $(n=39)$ or contextual fear training $(n=22)$ as described above. The rats were then returned to their home cage and killed 10,30, or $60 \mathrm{~min}$ later. Ten of the rats killed at $60 \mathrm{~min}$ had received an immediate posttraining injection of RAP $(5 \mu \mathrm{g} / \mu \mathrm{l})$ or DMSO into the amygdala. Rats that underwent contextual fear training were all killed 60 min after training. Separate groups of untrained rats were killed from their home cage. Brains were removed and immediately put on dry ice and stored in a $-80^{\circ} \mathrm{C}$ freezer before dissection. Amygdala tissue was dissected out by blocking the brain in a rat brain matrix (Harvard Apparatus, Holliston, MA) and making a single coronal cut at the anterior tip of the amygdala and one at the posterior end of the amygdala. Both sides of the whole amygdala were dissected out from the blocked tissue by making a cut along the external capsule and a diagonal cut along the optic tract. The tissue sample was homogenized in buffer (all in $100 \mathrm{ml}$ DDH20; 0.605 g Tris- $\mathrm{HCl}, 0.25$ g sodium deoxycholate, $0.876 \mathrm{~g} \mathrm{NaCl}$, $0.038 \mathrm{~g}$ EDTA, $0.0042 \mathrm{~g} \mathrm{NaF}, 1 \mu \mathrm{g} / \mathrm{ml}$ PMSF, $1 \mu \mathrm{g} / \mathrm{ml}$ leupeptin, $1 \mu \mathrm{g} / \mathrm{ml}$ aprotinin, $10 \mathrm{ml}$ 10\% SDS, $1 \mathrm{~mm}$ sodium orthovanadate) and immediately placed on dry ice. Samples were stored at $-80^{\circ} \mathrm{C}$ until needed. Samples were thawed and then centrifuged at $4000 \mathrm{rpm}$ for $20 \mathrm{~min}$; the supernatant was removed and measured using a Bradford protein assay kit (Bio-Rad, Hercules, CA). Protein samples were normalized and loaded on $6 \%$ SDS-PAGE. Proteins were transferred from the gel to a membrane using a semidry transfer apparatus (Bio-Rad). Membranes were incubated in blocking buffer for $2 \mathrm{~h}$ and then incubated overnight at $4^{\circ} \mathrm{C}$ in primary antibody for phospho-p70s6K (Thr 412) (1:1000; Upstate Biotechnology, Lake Placid, NY) or nonphospho-p70s6K (1:1000; Upstate Biotechnology). After primary antibody exposure, the membranes were incubated in secondary antibody (1:5000; Upstate Biotechnology) for $90 \mathrm{~min}$. Membranes were washed thoroughly, placed in a chemiluminescence solution for 3 min (Santa Cruz Biotechnology, Santa Cruz, CA), and exposed to autoradiographic film (Hyperfilm MP). Images were taken and densitometry was performed using NIH Image J. A percentage of control score was derived for each rat by dividing each animal's relative optical density score by the home cage control group mean. This percentage of control score was then statistically analyzed using a one-way ANOVA and Fisher's least significant difference (LSD) post hoc tests.

Immunofluorescence procedure. After contextual fear training, the rats in this experiment $(n=3)$ were killed with an intraperitoneal injection of sodium pentobarbital $(100 \mathrm{mg} / \mathrm{kg})$ and perfused with saline followed by $10 \%$ buffered formaldehyde solution. Brains were quickly removed and stored overnight in $10 \%$ buffered formaldehyde. The following day, 
brains were washed three times with $0.1 \mathrm{M}$ PBS for $10 \mathrm{~min}$. Fifty micrometer sections were taken throughout the amygdala and placed in a 24-well plate filled with $500 \mu \mathrm{l}$ of $0.1 \mathrm{M}$ PBS. After sections were taken, slices were incubated on a titer plate in $500 \mu \mathrm{l}$ of $1 \% \mathrm{NaBH} 4$ for $15 \mathrm{~min}$, followed by two $10 \mathrm{~min}$ washes with $0.1 \mathrm{M}$ PBS, and a $30 \mathrm{~min}$ wash with $10 \%$ NGS. Slices were incubated overnight in $500 \mu \mathrm{l}$ of primary antibody diluted 1:50 against phospho-p70s6K (Thr 412) (Upstate Biotechnology). The next day, slices were removed from the refrigerator, rinsed in $0.1 \mathrm{M}$ PBS (two times, $10 \mathrm{~min}$ each), and incubated in anti-rabbit fluorescent secondary (1:200; Alexa Fluor 594; Invitrogen, Eugene, OR) in the dark for $2 \mathrm{~h}$. Slices were then rinsed in $0.1 \mathrm{~m}$ PBS (two times, $5 \mathrm{~min}$ each) and mounted onto untreated glass slides using Prolong Antifade mounting medium (Invitrogen). Photomicrographs from these slides were acquired using a microscope (Olympus, Center Valley, PA) equipped with epifluorescence capabilities. All images were acquired using the same parameters (exposure time, etc.), and all brightness and contrast adjustments were exactly the same for all of the images (see Fig. $4 c-e)$.

Autoradiography procedure. Five male Long Evans rats were prepared with bilateral cannulas aimed at the amygdala $(\mathrm{AP},-2.8 ; \mathrm{L}, \pm 5.0 ; \mathrm{V}$, $-7.2)$. Three rats received infusions of RAP $(1 \mu \mathrm{g} / \mu \mathrm{l} ; 0.5 \mu \mathrm{l} / \mathrm{side})$ into one side of the amygdala and DMSO in the other, while two rats received injections of anisomycin $(125 \mu \mathrm{g} / \mu \mathrm{l}$; Sigma) or artificial CSF (ACSF). Thirty minutes later, these rats received $1.0 \mu \mathrm{l} /$ side of $\left[\mathrm{U}_{-}{ }^{14} \mathrm{C}\right]$-leucine $(50 \mu \mathrm{Ci} / \mathrm{ml}, 306 \mathrm{mCi} / \mathrm{mmol}$ specific activity; GE Healthcare, Piscataway, $\mathrm{NJ}$ ) into both hemispheres. One hour later, all rats were killed and perfused with saline followed by $10 \%$ buffered formalin. Brains were stored overnight in sucrose formalin, and the following day the tissue was sectioned by taking $40 \mu \mathrm{m}$ slices beginning in the area $\sim 2 \mathrm{~mm}$ anterior to the amygdala and continuing to the area $\sim 2 \mathrm{~mm}$ posterior to the amygdala. Sections were mounted on slides and were then exposed to autoradiographic film (Hyperfilm MP Film; GE Healthcare) for 2 weeks. Densitometry was performed on developed films using an automated system (MCID; Imaging Research, St. Catharines, Ontario, Canada).

\section{Results}

\section{Rapamycin delivered into the amygdala disrupts the consolidation of fear memory}

In the first experiments, rats were trained in a fear-conditioning procedure by pairing white noise with footshock. Immediately after this training, rats were given infusions $(0.5 \mu \mathrm{l} /$ side in all cases) of DMSO or different doses of RAP (5-1000 $\mathrm{ng} / \mu \mathrm{l})$ into the amygdala. Results from an ANOVA on data from this experiment showed a dose-dependent effect of RAP on the consolidation of fear memory when animals were tested to the auditory CS $\left(F_{(5,32)}=2.698 ; p<0.05\right)$ and to the training context $\left(F_{(5,32)}=\right.$ $2.634 ; p<0.05) 24 \mathrm{~h}$ after training (Fig. $1 a, b)$. Tukey's honestly significant difference post hoc tests revealed a significant difference during the auditory CS test between controls and animals given $1 \mu \mathrm{g}$ of RAP. During the context test, post hoc analysis showed a significant difference between DMSO-infused rats and those given $500 \mathrm{ng}$ of RAP. No statistically significant differences were seen between DMSO controls and any of the other groups during testing. Likewise, no differences were observed during the training session or during the baseline period of the auditory CS test (data not shown).

In a subsequent experiment, rats were trained with a slightly different training procedure and were given infusions $(0.5 \mu \mathrm{l} / \mathrm{side}$ in all cases) of DMSO or a slightly higher dose or RAP $(5 \mu \mathrm{g} / \mu \mathrm{l})$ into the amygdala. An independent samples $t$ test performed on these data confirmed that rats infused with this dose of RAP showed significantly less freezing to both the training context $\left(t_{20}=2.373 ; p<0.05\right)$ and the auditory CS $\left(t_{20}=2.269 ; p<0.05\right)$ when tested $24 \mathrm{~h}$ later (Fig. $1 c, d$ ). No statistically reliable differences were seen at any other point during training or testing (data not shown).
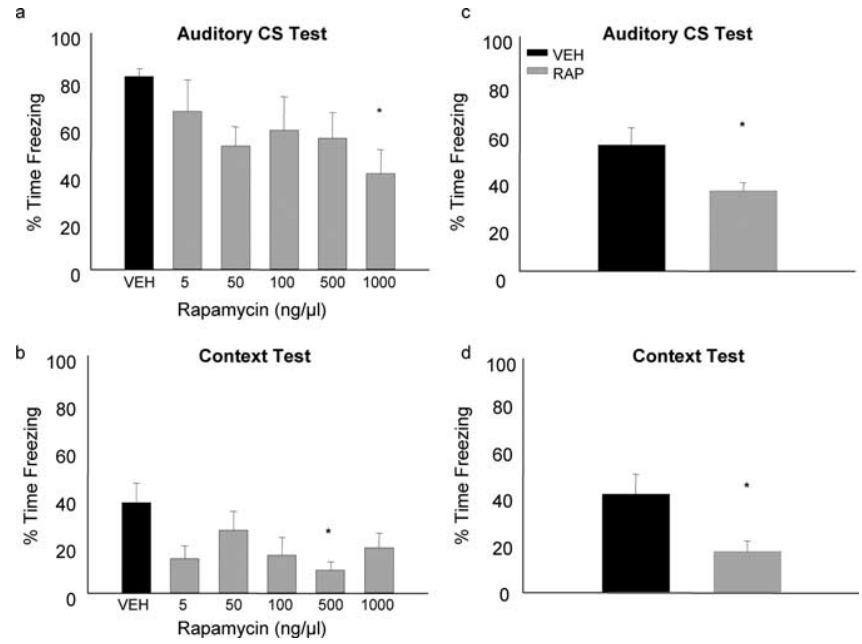

Figure 1. Consolidation of fear memory is dependent on rapamycin-sensitive signaling in the amygdala. Mean percentage of time spent freezing ( \pm SEM) during entire 15 min context test and 5 min auditory CS test is shown. $\boldsymbol{a}, \boldsymbol{b}$, Rats were administered different doses of RAP $(5-1000 \mathrm{ng} / \mu \mathrm{l})$ into the amygdala immediately after training and tested to the auditory $\mathrm{CS}(\boldsymbol{a})$ and training context $(\boldsymbol{b})$ the next day. A slightly different training protocol was used in the next experiment with a higher dose of RAP. $\boldsymbol{c}, \boldsymbol{d}$, Rats given $5 \mu \mathrm{g} / \mu$ l RAP showed significantly less freezing to both the auditory $\mathrm{CS}(\boldsymbol{c})$ and training context $(\boldsymbol{d})$ compared with controls $\left({ }^{*} p<\right.$ 0.05).

To rule out the possibility that RAP is disrupting memory by simply shutting down protein synthesis altogether, we looked at the effect of RAP on local [C14]-leucine incorporation in the amygdala. We observed only a minor decrease $(\sim 12 \%$ reduction from control) compared with that seen with a behaviorally effective dose of anisomycin ( $\sim 50 \%$ reduction from control) (Fig. $2 a, b)$. These data are consistent with other work showing only small changes in overall protein synthesis rates after RAP treatment (Jefferies et al., 1994), indicating that blockade of the mTOR pathway with RAP prevents the translation of a distinct subset of relevant transcripts instead of a global blockade of protein synthesis. The mechanism by which this specificity is achieved is not completely understood but is thought to involve preferential targeting by mTOR effectors of mRNA with extensive secondary structure and/or oligopyrimidine tracts in the 5' region (Raught et al., 2001).

\section{Fear conditioning activates the time-dependent phosphorylation of p70s6K}

The second set of experiments was designed to examine the effects of fear learning on the relative expression of phosphorylated and nonphosphorylated p70s6K using Western blot analysis. This kinase is believed to be activated by mTOR, and phosphorylation of p70s6K can be blocked by RAP (Jefferies et al., 1994). In this experiment, rats were trained in the same fear-conditioning procedure as described above and killed 10, 30, or 60 min after training. Controls were killed from their home cage without training. A one-way ANOVA performed on the data (Fig. 3a) showed a time-dependent change in the level of phospho-p70s6K in the amygdala as a result of conditioning $\left(F_{(3,25)}=10.358 ; p<\right.$ $0.05)$. Fisher's LSD post hoc tests revealed a significant increase in phospho-p70s6K levels versus home cage controls in rats killed at $30(p<0.05)$ and $60(p<0.01)$ minutes after training. The post hoc tests also showed that tissue taken from rats killed at 30 and 60 min after training showed significantly more phospho-p70s6K expression than the group killed at $10 \mathrm{~min}$. The same tissue samples were also assayed for changes in total p70s6K. Importantly, a 
a

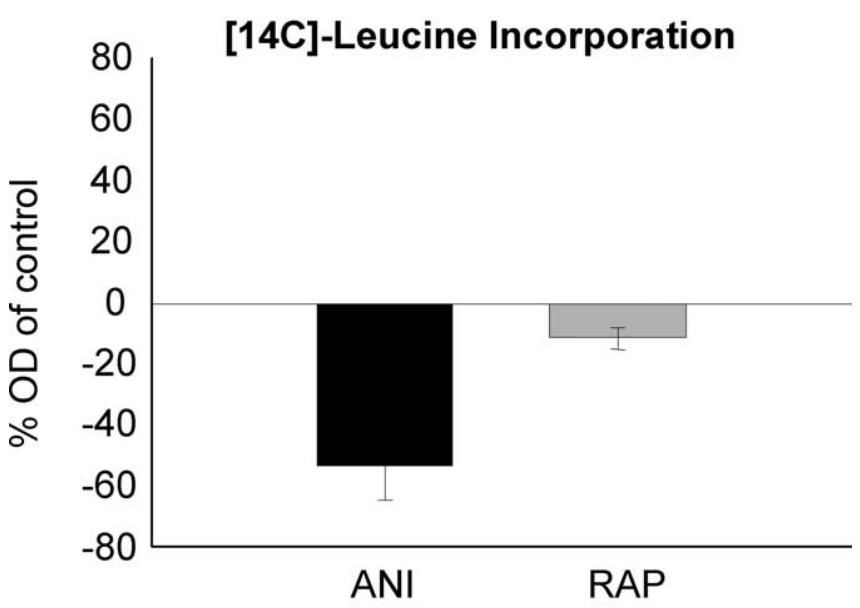

b
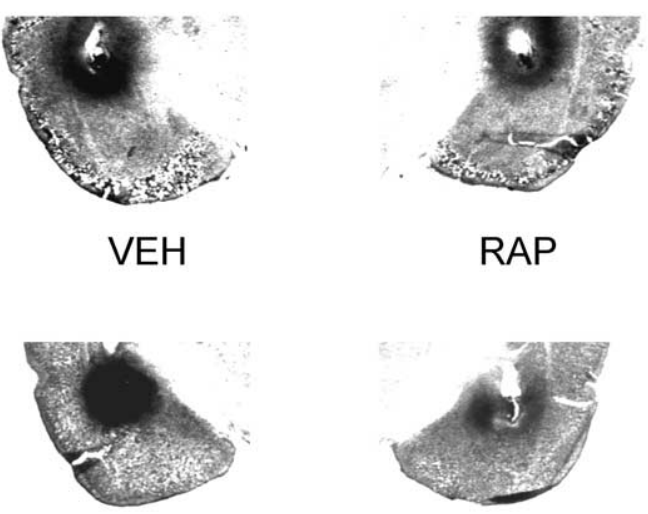

ACSF

ANI

Figure 2. Intra-amygdala infusion of rapamycin does not block global rates of translation. $\boldsymbol{a}$, As measured by $\left[{ }^{14} \mathrm{C}\right]$-leucine incorporation, RAP reduces global rates of protein synthesis only slightly compared with anisomycin (ANI). $\boldsymbol{b}$, Overlay of representative autoradiographic images on Nissl-stained brain sections for tissue treated with RAP/VEH or ANI/ACSF. OD, Optical density. Error bars represent \pm SEM.

one-way ANOVA showed no significant changes in the total amount of p70s6K because of training condition (Fig. 3c).

\section{Rapamycin blocks training-related increases \\ in phospho-p70s6K}

Data from the first experiment showed that an immediate posttraining injection of RAP in the amygdala disrupts the formation of fear memory. We also showed that a downstream target of the mTOR kinase phospho-p70s6K increases in a time-dependent manner after fear learning. To link these two observations, we next examined the effect of immediate posttraining infusions of RAP $(5 \mu \mathrm{g} / \mu \mathrm{l})$ in the amygdala on the increase in phosphop70s6K seen at $60 \mathrm{~min}$ after training. A one-way ANOVA on the data from this experiment showed that animals given RAP after training showed less phospho-p70s6K expression in the amygdala at 60 min compared with rats infused with vehicle $(\mathrm{VEH})\left(F_{(1,9)}=\right.$ $6.630 ; p<0.05$ ) (Fig. 3b). An ANOVA confirmed that no differences were seen in total p70s6 K expression in the amygdala after treatment with RAP (Fig. 3d). The fact that a memory-impairing dose of RAP also blocked the training-related changes in
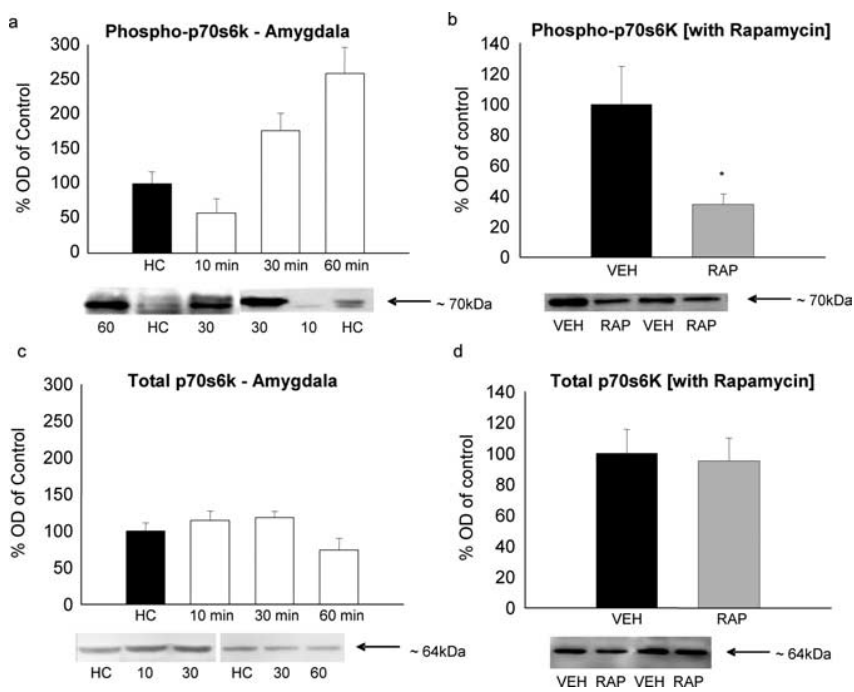

Figure 3. Time-dependent activation of phospho-p70s6K in the amygdala after fear conditioning. Bars represent mean optical density (OD) values ( \pm SEM) expressed as a percentage of control. $\boldsymbol{a}$, Rats killed at 30 and $60 \mathrm{~min}$ after fear conditioning showed significantly more phospho-p70s6K expression in the amygdala compared with home cage $(\mathrm{HC})$ controls or animals killed $10 \mathrm{~min}$ after conditioning. This increase in phospho-p70s6K seen $60 \mathrm{~min}$ after training is reduced by an immediate posttraining injection of $\operatorname{RAP}(\boldsymbol{b}) . \boldsymbol{c}, \boldsymbol{d}$, No differences between groups were observed when the same samples were probed with an antibody for total p70s6K

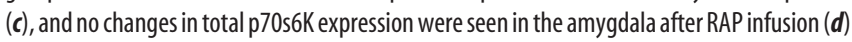
$\left({ }^{*} p<0.05\right)$.

phospho-p70s6K suggests that the observed increase in the activity of this enzyme is related to memory consolidation.

Training-related increase in phospho-p70s6 $\mathrm{K}$ is specific to the learned association

We exposed rats to contextual fear conditioning or an associative control procedure in which shock is delivered immediately after placement in the chamber. This procedure equates exposure to the shock and environmental cues but does not support fear learning or memory formation (Fanselow, 1990). Rats were killed 60 min later and processed for Western blots or immunofluorescence. A one-way ANOVA on data from the Western blot experiment confirmed that expression of total p70s6K in the amygdala was similar in trained animals when compared with controls and nonstimulated (home cage) rats $(F<1.0)$ (Fig. $4 a)$, although there was a significant change in phospho-p70s6K levels with this procedure $\left(F_{(2,19)}=4.485 ; p<0.05\right)$. Fisher's LSD post hoc tests showed that significant increases in immunoreactivity to phosphorylated p70s6k was seen only in the amygdala of animals exposed to the procedure that results in robust learning (delay vs home cage, $p<0.01$ ) and was essentially absent in controls (Fig. $4 b)$. The immunofluorescence images show the same pattern as the Western blot data whereby dense staining for phosphop70s6 $\mathrm{K}$ is only seen in the lateral amygdala from the rat exposed to a delayed shock procedure (Fig. $4 c-e$ ).

\section{Postretrieval infusion of Rapamycin disrupts memory reconsolidation}

To determine whether the mTOR pathway is involved in the long-term stability of fear memory, we gave rats postretrieval infusions of RAP or VEH into the amygdala. A one-way ANOVA performed on the data collected during the test session (Fig. 5) showed that rats treated with RAP after retrieval showed significantly less freezing when the memory was tested the next day 

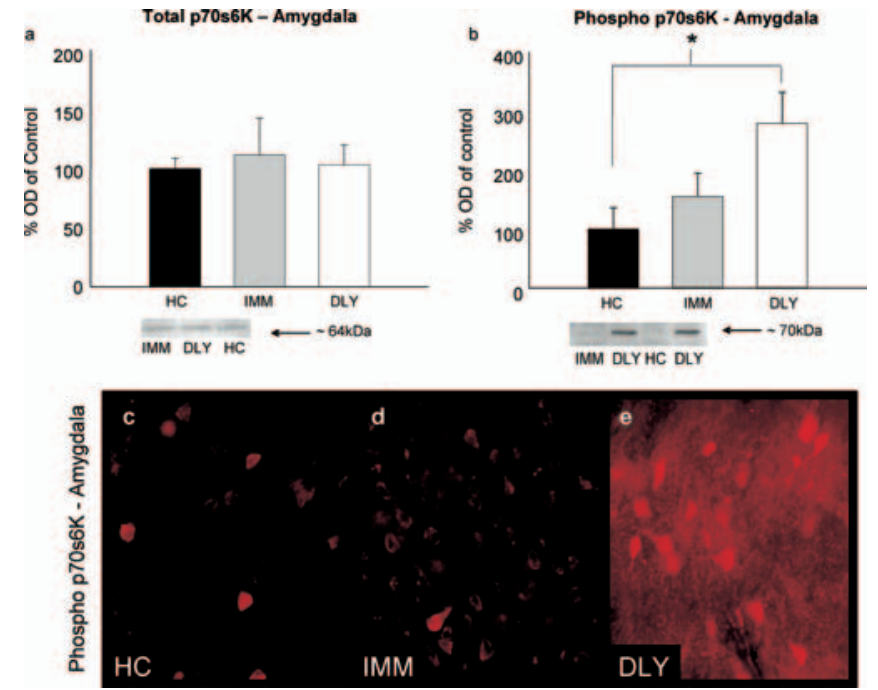

Figure 4. Training-related increase in phospho-p70s6K is specific to the learned association. Bars represent mean optical density (OD) values ( \pm SEM) expressed as a percentage of control. $\boldsymbol{a}, \boldsymbol{b}$, There were no changes in total-p70s6K expression after immediate or delayed shock (a), but a significant increase in phospho-p70s6K was seen only in rats that received a delayed shock (b). Immunofluorescence images ( $20 \times$ magnification) from single subjects killed from their home cage $(\mathrm{HC})(\boldsymbol{c})$ or given a single shock immediately (IMM) or 2 min after placement into the chamber (DLY) are shown. $\boldsymbol{d}, \boldsymbol{e}$, Although controls $(\boldsymbol{d})$ received the same shock and stimulus exposure, phosphorylation of the enzyme in the lateral amygdala was seen only in rats that formed new long-term memory for fear conditioning $(e)\left({ }^{*} p<0.05\right)$.

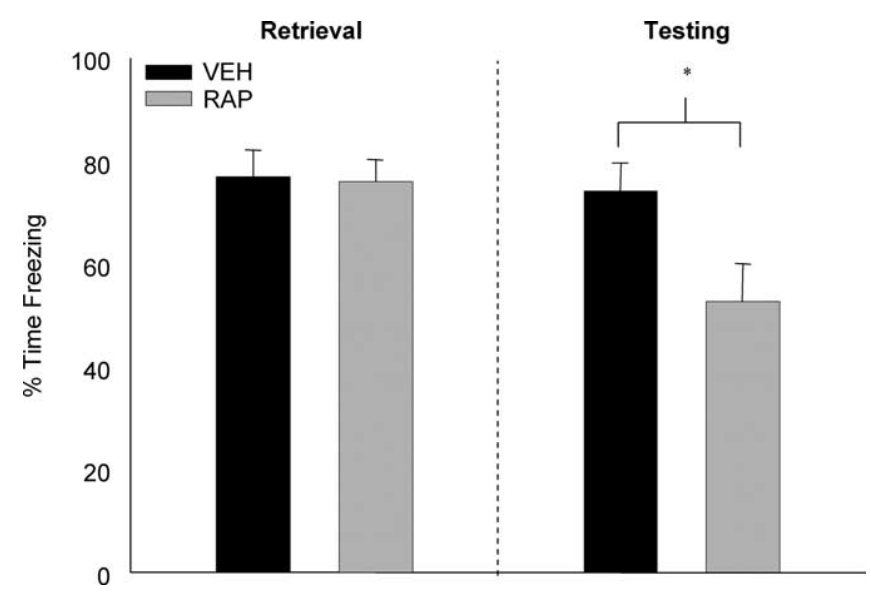

Figure 5. Infusion of rapamycin into the amygdala after memory retrieval disrupts reconsolidation. Mean percentage of time spent freezing ( \pm SEM) during the $32 \mathrm{~s}$ retrieval session and 2 min test is shown. Rats given RAP (gray bars) into the amygdala immediately after retrieval showed less freezing when tested the next day compared with rats that received VEH (black bars) infusion $\left({ }^{*} p<0.05\right)$.

$\left(F_{(1,21)}=5.444 ; p<0.05\right)$. There were no significant differences at any other point during training, retrieval, or testing ( $F$ values, all $<1.7)$. These data argue that, in addition to being critical for initial consolidation, mTOR signaling is also important for the reconsolidation of fear memory after retrieval.

\section{Discussion}

Despite considerable evidence that RAP-sensitive signaling is involved in synaptic plasticity (Casadio et al., 1999; Tang et al., 2002), very few studies have examined the effects of RAP on learning and memory in behaving animals. The data from our experiments show that consolidation of fear memory requires
RAP-sensitive signaling in the amygdala. Rats infused with RAP immediately after training exhibited decreased freezing to the training context and to the discrete auditory CS. We also observed an increase in amygdalar phospho-p70s6K at 30 and 60 min after training. The increase in phospho-p70s6K seen at 60 min was blocked by a memory-impairing dose of RAP. Finally, we showed that mTOR signaling might also be important for memory reconsolidation as RAP given into the amygdala after a reminder treatment disrupted fear memory.

Two key questions regarding the role of the mTOR-signaling pathway in synaptic plasticity and memory are as follows: (1) what rapamycin-sensitive proteins are involved, and (2) in which part(s) of the neuron are they located. The mTOR pathway is known to regulate the translation of proteins that make up translational machinery, including various elongation factors and ribosomal proteins (Meyuhas, 2000). Rapamycin-sensitive signaling has been shown to be involved in BDNF-mediated activation of translational factors and increases in selected proteins. Takei et al. (2001) showed that BDNF induces the expression of phospho$4 \mathrm{E}-\mathrm{BP} 1$ and phospho-eIF4E in cortical neurons, and that this effect can be blocked by RAP. A related study found that RAP also attenuated the BDNF-induced expression of Arc and CamkII protein in cortical neurons (Takei et al., 2004). Both of these proteins have been shown to be involved in synaptic plasticity and memory formation (Silva et al., 1992; Guzowski et al., 2000). Takei et al. (2004) also showed that Arc and CamkII are expressed dendritically in a RAP-sensitive manner. Furthermore, other research has shown that BDNF-mediated increases in dendritic synthesis of homer2 during neural development can also be blocked by pretreatment with RAP (Schratt et al., 2004). Work focusing on the molecular basis of LTP has also suggested that RAP has selective effects on dendritically located transcripts. For example, previous work has shown that LTP-induced expression of phospho-p70s6 $\mathrm{K}$ is enhanced selectively in dendritic areas in a RAP-dependent manner in CA1 neurons (Cammalleri et al., 2003). Other work has shown RAP-sensitive local protein synthesis to be necessary for LTP in hippocampal neurons that have been severed from their cell bodies (Cracco et al., 2005). Collectively, these studies suggest that the mTOR pathway regulates the expression of several proteins that have a key role in synaptic plasticity, and that this regulatory function of mTOR is attributable to, in part, the disruption of specific translational events occurring at dendritic sites. However, the effects of RAP do not appear to be entirely restricted to dendrites as some evidence (Takei et al., 2004) has shown that RAP also has effects on somatic protein synthesis.

One of the more interesting observations in our data was that RAP disrupted memory reconsolidation. Several studies have shown that fear memory is susceptible to disruption with protein synthesis inhibitors around the time of retrieval (Nader et al., 2000; Kida et al., 2002). Subsequent work has examined whether memory reconsolidation shares the same molecular mechanisms as initial consolidation. In particular, there is some controversy over whether memory reconsolidation requires transcription. Some reports have argued that like initial consolidation, memory reconsolidation relies on the synthesis of new mRNA and various transcription factors (Kida et al., 2002; Sangha et al., 2003; Lee et al., 2004). However, under some conditions, transcription does not appear to be involved in memory reconsolidation. Recent data from our laboratory showed that whereas amygdalar protein synthesis is necessary for the reconsolidation of fear memory, mRNA synthesis is not (Parsons et al., 2006). These data suggest that under some conditions, the synthesis of new mRNA is not 
needed for reconsolidation, and that the protein needed for memory reconsolidation might be made from dendritically located mRNA stores. This conclusion is supported by the recent finding that memory consolidation in the chick can be disrupted by disabling the neuronal transport mechanism. Interestingly, the very same treatment had no effect after a reminder treatment arguing that synaptic strengthening that supports memory reconsolidation in this preparation depends on proteins synthesized in dendrites and does not require somatic protein synthesis (Mileusnic et al., 2005). As we outlined above, there is considerable evidence that many of the effects of mTOR on plasticity are localized to dendrites. The positive effect with RAP on reconsolidation in our study suggests that the mTOR pathway may be involved in regulating the local protein synthesis that supports memory reconsolidation, but additional work would be needed to strengthen the link between the mTOR pathway and dendritic protein synthesis in our preparation to make this conclusion.

The data from this study add to a growing body of work arguing for a role of specific translational regulatory processes in synaptic plasticity and learning and memory. In a series of experiments using genetically altered mice, Kelleher et al. (2004) showed that the ERK pathway is involved in the regulation of protein synthesis through various translation factors, including the eukaryotic initiation factor $4 \mathrm{E}, 4 \mathrm{E}$ binding protein-1, and $\mathrm{S} 6$ ribosomal protein. Interestingly, in this same study, the phosphorylation of all of these factors was shown to be sensitive to rapamycin treatment. These same mice showed deficits on several measures of synaptic plasticity and memory. Previous work has shown that ERK signaling in the amygdala is critical for the consolidation and reconsolidation of fear memory (Schafe et al., 2000; Duvarci et al., 2005). The role of ERK in synaptic plasticity and memory formation has been ascribed primarily to the effects it has on the regulation of transcription (Impey et al., 1998). However, given that ERK signaling is also known to affect translational processes, it is difficult to determine whether the effects that ERK inhibition have on memory are a result of regulation of transcription or translation. Our results with rapamycin indicate that the memory-impairing effects of ERK inhibitors could essentially be explained in terms of their effects on the translational pathway controlled by mTOR.

The behavioral effects of rapamycin on memory in this study could be considered partial as evidenced by the fact that rats treated with RAP still show some freezing behavior. However, the effect sizes with RAP-induced memory impairment are generally similar to experiments in which we have blocked more global cellular processes (i.e., protein or mRNA synthesis) in the amygdala with very similar behavioral training protocols (Parsons et al., 2006). To the extent to which the effects are partial, it is possible that any residual freezing is mediated by other intracellular mechanisms operating in parallel. Another possibility is that although the expression of phospho-p70s6K is highly reduced in the amygdala after RAP treatment, it is not a total blockade, and therefore the residual freezing might be supported by any kinase activity that was not affected.

Data from the current set of experiments show that consolidation and reconsolidation of fear memory is sensitive to RAP treatment in the amygdala. Furthermore, we observed timedependent changes in phophso-p70s6K after conditioning, which were demonstrated to be sensitive to RAP. The observation that activity of the mTOR pathway is involved in the consolidation of fear memory adds to an emerging body of data (Kelleher et al., 2004; Costa-Mattioli et al., 2005) that translational control is crucial for the regulation of synaptic plasticity and memory consolidation.

\section{References}

Bailey DJ, Kim JJ, Sun W, Thompson RF, Helmstetter FJ (1999) Acquisition of fear conditioning in rats requires the synthesis of mRNA in the amygdala. Behav Neurosci 113:276-282.

Banko JL, Poulin F, Hou L, DeMaria CT, Sonenberg N, Klann E (2005) The translation repressor 4E-BP2 is critical for eIF4F complex formation, synaptic plasticity, and memory in the hippocampus. J Neurosci 25:9581-9590.

Cammalleri M, Lutjens R, Berton F, King AR, Simpson C, Francesconi W, Sanna PP (2003) Time-restricted role for dendritic activation of the mTOR-p70S6K pathway in the induction of late-phase long-term potentiation in the CA1. Proc Natl Acad Sci USA 100:14368-14373.

Casadio A, Martin KC, Giustetto M, Zhu H, Chen M, Bartsch D, Bailey CH, Kandel ER (1999) A transient, neuron-wide form of CREB-mediated long-term facilitation can be stabilized at specific synapses by local protein synthesis. Cell 99:221-237.

Costa-Mattioli M, Gobert D, Harding H, Herdy B, Azzi M, Bruno M, Bidinosti M, Ben Mamou C, Marcinkiewicz E, Yoshida M, Imataka H, Cuello AC, Seidah N, Sossin W, Lacaille JC, Ron D, Nader K, Sonenberg N (2005) Translational control of hippocampal synaptic plasticity and memory by the eIF2alpha kinase GCN2. Nature 436:1166-1173.

Cracco JB, Serrano P, Moskowitz SI, Bergold PJ, Sacktor TC (2005) Protein synthesis-dependent LTP in isolated dendrites of CA1 pyramidal cells. Hippocampus 15:551-556.

Duvarci S, Nader K, LeDoux JE (2005) Activation of extracellular signalregulated kinase-mitogen-activated protein kinase cascade in the amygdala is required for memory reconsolidation of auditory fear conditioning. Eur J Neurosci 21:283-289.

Fanselow MS (1990) Factors governing one-trial contextual conditioning. Animal Learn Behav 18:264-270.

Fanselow MS, LeDoux JE (1999) Why we think plasticity underlying pavlovian fear conditioning occurs in the basolateral amygdala. Neuron 23:229-232.

Guzowski JF, Lyford GL, Stevenson GD, Houston FP, McGaugh JL, Worley PF, Barnes CA (2000) Inhibition of activity-dependent arc protein expression in the rat hippocampus impairs the maintenance of long-term potentiation and the consolidation of long-term memory. J Neurosci 20:3993-4001.

Impey S, Obrietan K, Wong ST, Poser S, Yano S, Wayman G, Deloulme JC, Chan G, Storm DR (1998) Cross talk between ERK and PKA is required for Ca2 + stimulation of CREB-dependent transcription and ERK nuclear translocation. Neuron 21:869-883.

Jefferies HB, Reinhard C, Kozma SC, Thomas G (1994) Rapamycin selectively represses translation of the "polypyrimidine tract" mRNA family. Proc Natl Acad Sci USA 91:4441-4445.

Josselyn SA, Shi C, Carlezon Jr WA, Neve RL, Nestler EJ, Davis M (2001) Long-term memory is facilitated by cAMP response element-binding protein overexpression in the amygdala. J Neurosci 21:2404-2412.

Kelleher RJ, III, Govindarajan A, Jung HY, Kang H, Tonegawa S (2004) Translational control by MAPK signaling in long-term synaptic plasticity and memory. Cell 116:467-479.

Kida S, Josselyn SA, de Ortiz SP, Kogan JH, Chevere I, Masushige S, Silva AJ (2002) CREB required for the stability of new and reactivated fear memories. Nat Neurosci 5:348-355.

Lee JL, Everitt BJ, Thomas KL (2004) Independent cellular processes for hippocampal memory consolidation and reconsolidation. Science 304:839-843.

Meyuhas O (2000) Synthesis of the translational apparatus is regulated at the translational level. Eur J Biochem 267:6321-6330.

Mileusnic R, Lancashire CL, Rose SP (2005) Recalling an aversive experience by day-old chicks is not dependent on somatic protein synthesis. Learn Mem 12:615-619.

Nader K, Schafe GE, LeDoux JE (2000) Fear memories require protein synthesis in the amygdala for reconsolidation after retrieval. Nature 406:722-726.

Parsons RG, Gafford GM, Baruch DE, Riedner BA, Helmstetter FJ (2006) Long-term stability of fear memory depends on the synthesis of protein but not mRNA in the amygdala. Eur J Neurosci 23:1853-1859. 
Paxinos G, Watson C (1998) The rat brain in stereotaxic coordinates, Ed 4. San Diego: Academic.

Raught B, Gingras AC, Sonenberg N (2001) The target of rapamycin (TOR) proteins. Proc Natl Acad Sci USA 98:7037-7044.

Sangha S, Scheibenstock A, Lukowiak K (2003) Reconsolidation of a longterm memory in Lymnaea requires new protein and RNA synthesis and the soma of right pedal dorsal 1. J Neurosci 23:8034-8040.

Schafe GE, LeDoux JE (2000) Memory consolidation of auditory pavlovian fear conditioning requires protein synthesis and protein kinase $\mathrm{A}$ in the amygdala. J Neurosci 20:RC96(1-5).

Schafe GE, Atkins CM, Swank MW, Bauer EP, Sweatt JD, LeDoux JE (2000) Activation of ERK/MAP kinase in the amygdala is required for memory consolidation of pavlovian fear conditioning. J Neurosci 20:8177-8187.

Schratt GM, Nigh EA, Chen WG, Hu L, Greenberg ME (2004) BDNF regulates the translation of a select group of mRNAs by a mammalian target of rapamycin-phosphatidylinositol 3-kinase-dependent pathway during neuronal development. J Neurosci 24:7366-7377.

Silva AJ, Paylor R, Wehner JM, Tonegawa S (1992) Impaired spatial learn- ing in alpha-calcium-calmodulin kinase II mutant mice. Science 257:206-211.

Takei N, Kawamura M, Hara K, Yonezawa K, Nawa H (2001) Brain-derived neurotrophic factor enhances neuronal translation by activating multiple initiation processes: comparison with the effects of insulin. J Biol Chem 276:42818-42825.

Takei N, Inamura N, Kawamura M, Namba H, Hara K, Yonezawa K, Nawa H (2004) Brain-derived neurotrophic factor induces mammalian target of rapamycin-dependent local activation of translation machinery and protein synthesis in neuronal dendrites. J Neurosci 24:9760-9769.

Tang SJ, Reis G, Kang H, Gingras AC, Sonenberg N, Schuman EM (2002) A rapamycin-sensitive signaling pathway contributes to long-term synaptic plasticity in the hippocampus. Proc Natl Acad Sci USA 99:467-472.

Tischmeyer W, Schicknick H, Kraus M, Seidenbecher CI, Staak S, Scheich H, Gundelfinger ED (2003) Rapamycin-sensitive signalling in long-term consolidation of auditory cortex-dependent memory. Eur J Neurosci 18: 942-950. 